Cahiers de philosophie de l'université de

Totalitas. Aux origines d'un concept

\title{
Totum et totalitas dans la première école franciscaine
}

\section{Laure Solignac}

\section{(2) OpenEdition}

1 Journals

Édition électronique

URL : https://journals.openedition.org/cpuc/341

DOI : 10.4000/cpuc.341

ISSN : 2677-6529

Éditeur

Presses universitaires de Caen

\section{Édition imprimée}

Date de publication : 31 décembre 2016

Pagination : 77-94

ISBN : 978-2-84133-842-9

ISSN : $1282-6545$

\section{Référence électronique}

Laure Solignac, «Totum et totalitas dans la première école franciscaine », Cahiers de philosophie de l'université de Caen [En ligne], 53 | 2016, mis en ligne le 01 février 2019, consulté le 31 janvier 2023. URL : http://journals.openedition.org/cpuc/341 ; DOI : https://doi.org/10.4000/cpuc.341

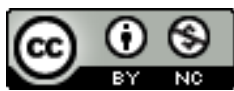

Creative Commons - Attribution - Pas d'Utilisation Commerciale 4.0 International - CC BY-NC 4.0 https://creativecommons.org/licenses/by-nc/4.0/ 


\section{Totum et totalitas dans la première école franciscaine}

D ANS La Relation CRITIQUe, Jean Starobinski évoque la lassitude, voire le dégoût, que lui inspirent les "machines à produire de l'Un", en l'occurrence les théories littéraires en vigueur dans les années 1960, mais plus largement toute pensée ayant une prétention à la fois systématique et totalisante ${ }^{1}$. Une vingtaine d'années plus tard, à la fin des années 1980 et dans un tout autre contexte, Pierre Aubenque écrit un célèbre article sur le concept d'analogie, dans les Études philosophiques, où il analyse et dénonce à la fois la manière dont ce concept, conçu pour rendre compte de multiplicités irréductibles et hétérogènes, a été métamorphosé par les néoplatoniciens en vue de la réduction de toutes choses à une unité première, qui vient ainsi reléguer au second plan, voire annuler leur multiplicité réelle et si passionnante pour le spécialiste d'Aristote ${ }^{2}$.

Dans les deux cas, le processus de totalisation apparaît comme une menace pour la multiplicité et la singularité, comme si les pensées de la totalité étaient essentiellement appauvrissantes et ennuyeuses, incapables en tout cas d'assumer la complexité et la diversité du réel. On peut y voir bien sûr la confirmation d'une décision philosophique importante qui caractérise notre temps: celle du privilège accordé à l'autre plutôt qu'au même, au multiple plutôt qu'à l'un, à la diversité plutôt qu'à l'universel. On croit ainsi avoir pris le parti du réel contre son arraisonnement possible et contre son assujettissement.

Mais dans ce jugement sévère sur la totalité, nous pouvons également voir le signe d'un appauvrissement considérable de notre pensée au sujet du tout et de la totalité, et en ce sens, la lecture des auteurs médiévaux est des plus instructives pour nous. En effet, du fait des exigences maximales

1. J. Starobinski, La Relation critique, $2^{\mathrm{e}}$ éd., Paris, Gallimard, 2001, p. 49.

2. P. Aubenque, «Sur la naissance de la doctrine pseudo-aristotélicienne de l'analogie de l'être», Les Études philosophiques, $\mathrm{n}^{\circ} 3-4,1989$, p. 304. 
de la théologie trinitaire pour la raison, mais aussi du fait de l'absence d'un cadre totalisant univoque, les penseurs médiévaux, à la suite de Proclus et de Denys l'Aréopagite, ont su élaborer non seulement une conception polyphonique du tout et de la totalité, au service des êtres réels et de leurs correspondances secrètes, mais aussi une conception hospitalière et humble de la totalité à l'égard de la multiplicité et de la différence. Et s'ils y sont parvenus, pourquoi ne le pourrions-nous pas?

Il s'agit simplement dans les pages qui suivent de montrer que la première école franciscaine de Paris, dans les années 1240 et 1250, constitue un bon exemple de cette possible et généreuse discrétion de la totalité. Plus précisément, à partir d'extraits de la Summa Halensis et de différentes œuvres de saint Bonaventure, il s'agit de mettre au jour trois faits qui méritent notre attention: $1 /$ chez les premiers penseurs franciscains apparaît ou plutôt réapparaît une façon de concevoir le tout indépendamment de toute composition et de toute partie, et cela non pas sur le terrain de la logique, mais sur celui de la théologie ; 2 / malgré cette conception très exigeante du tout comme totalité, l'un d'entre eux, à savoir Bonaventure, trouve tout de même le moyen de décliner cette totalité sur plusieurs niveaux, celui de l'homme et celui du monde, d'une manière qui n'est pas purement rhétorique et sans que ces différents niveaux de totalité se réduisent les uns aux autres; 3 / enfin, la totalité n'est pas elle-même une notion totalisante: elle fonctionne et trouve son sens avec d'autres concepts; le tout, bien sûr, mais aussi la perfection, l'éternité, la présence, ou encore la forme substantielle.

\section{Les lieux stratégiques de la conceptualisation du tout et de la totalité dans la Summa Halensis}

La Summa Halensis est une œuvre collective dont le maître d'œuvre fut Alexandre de Halès lui-même, et à laquelle participèrent probablement Jean de la Rochelle, Guillaume de Méliton et Eudes Rigaud. Cette œuvre monumentale donne d'utiles points de repère sur les problèmes traités et les concepts utilisés au milieu du XIII ${ }^{\mathrm{e}}$ siècle, juste avant l'extraordinaire floraison de la seconde moitié du XIII ${ }^{\mathrm{e}}$ siècle, avec les sommes de Thomas d'Aquin et les œuvres de Bonaventure. En ce sens, la Summa Halensis joue pour la lignée franciscaine un rôle analogue à celui d'Albert le Grand pour la lignée dominicaine. Dans la Summa, les mises au point sur le totum apparaissent dans trois contextes principaux: la théologie (relativement à l'essence divine, et non à la Trinité), la cosmologie et dans une moindre mesure, l'anthropologie. Il semble que le mot totalitas ne soit pas utilisé par les rédacteurs de la Summa, qui préfèrent distinguer différentes sortes 
de «tout». Mais l'absence du mot ne signifie pas nécessairement l'absence de la chose.

Parcourons rapidement les trois étapes où se révèle la manière dont les rédacteurs de la Summa pensent le tout. La première étape n'est autre que la définition de l'éternité 3 , qui fait partie du traité sur l'immensité de l'essence divine, lequel vient juste à la suite du traité sur la simplicité divine, où il n'est pas question de la totalité - alors que Bonaventure ne cessera de montrer la fécondité du lien entre ces deux concepts. La définition de l'éternité passe par la juste compréhension d'une formule de Boèce à la fin de son De consolatione Philosophiae: l'éternité est «la possession parfaite, totale et simultanée (tota simul) d'une vie sans terme " ${ }^{4}$; autrement dit, Dieu est tout entier dans chaque instant. L'exégèse de cette formule fournit l'occasion d'une distinction entre une définition positive du tout (position d'un tout constitué de parties - secundum positionem constitutum ex partibus) et une définition négative du tout (négation de la diminution ou de la limite - per abnegationem diminutionis) : ce dont rien n'est au-dehors (cui nihil est extra), ce qui n'est par conséquent limité ou diminué par rien $^{5}$. De toute évidence, la source de cette définition négative se trouve dans le troisième livre de la Physique d'Aristote, auquel il faudra revenir. C'est précisément en ce second sens qu'il faut entendre la formule de Boèce afin qu'elle acquière un sens théologique: on peut dire que Dieu est totus simul, au sens où rien ne vient le limiter du dehors, dans la mesure où rien ne s'oppose à son immensité. Ainsi, dans sa version négative, la définition du tout coïncide avec la définition du caractère non circonscriptible et de l'infinité (non plus au sens aristotélicien, cette fois); en ce sens, cette définition peut être utilisée pour décrire l'être divin. Elle agit comme une définition possible de l'indéfinissable.

La deuxième étape arrive peu après. Au $\$ 71$, la question se précise: elle est de savoir si la nature divine est «totalement en tout temps et toujours» (utrum divina natura tota sit omni tempore et semper). Cette fois, les rédacteurs de

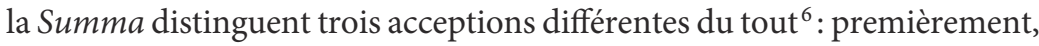

3. Alexandre de Halès, Summa theologica, t. I, $\$ 59$, PP. del Collegio S. Bonaventura (éd.), Quaracchi, Ad Claras Aquas, 1924, p. 87-89.

4. Boèce, De consolatione philosophiae, V, 6-4, C. Moreschini (éd.), Munich - Leipzig, De Gruyter (Bibliotheca scriptorum Graecorum et Romanorum Teubneriana), p. 155.

5. Alexandre de Halès, Summa theologica, t. I, $\$ 59$, resp. ad arg. 3, p. 87-89: "Ad tertium dicendum quod totum dicitur secundum positionem constitutum ex partibus: et hoc modo non accipitur totum; alio modo totum dicitur per abnegationem diminutionis, ut totum dicat non-diminitum vel cui nihil est extra: et hoc modo accipitur totum ".

6. Ibid., $\$ 71$, resp. ad arg. 2, p. 111 : «Ad secundum iam patet responsio, quod cum totum dicatur multipliciter - uno modo totum ex partibus constitutum; alio modo quod sic est ut nihil eius sit extra; tertio modo quod est perfectum et completum - hoc ultimo modo dicitur divina 
le tout comme ensemble constitué de parties (ce qui correspond au totum quantitativum chez Albert le Grand 7 ) - c'est le premier sens du tout dans le De divisione de Boèce, à savoir le totum continuum ${ }^{8}$; deuxièmement, le tout comme ce qui n'a pas d'extériorité (nous retrouvons la définition aristotélicienne du tout dans la Physique); troisièmement, le tout comme ce qui est "parfait et complet» (quod est perfectum et completum). Là encore, la source aristotélicienne semble l'emporter sur le De divisione de Boèce, d'autant plus qu'on ne trouve aucune allusion au totum gregem et au totum universale (respectivement les deuxième et troisième sens du tout selon Boèce). On peut toutefois se demander si ce «tout parfait» n'inclut pas en quelque manière le quatrième sens du tout chez Boèce, à savoir le tout de puissance9. Quoi qu'il en soit, seul ce troisième sens du tout comme perfectum et completum est considéré comme véritablement utile dans l'appréhension de la nature divine, comme si le second, cette fois, était insuffisant: «c'est en ce dernier sens que l'on dit de la nature divine qu'elle est totalement en tout lieu et en tout temps, parce qu'elle est parfaite et complète ${ }^{10}$. Pourquoi la définition négative passe-t-elle au second plan? On peut émettre l'hypothèse qu'il s'agit de la même définition, considérée sous deux angles différents; mais on ne peut précisément la vérifier qu'en allant à la source aristotélicienne commune de ces deux définitions du tout (le tout sans extériorité et le tout parfait). Si on se reporte à la Physique ${ }^{11}$, on constate qu'Aristote distingue l'infini et la totalité: l'infini "n'est pas ce qui n'a rien à l'extérieur de soi, mais c'est ce dont quelque chose est toujours à l'extérieur de lui » parce qu'il n'est pas complet. Au contraire:

Ce dont rien n'est à l'extérieur de lui, cela est achevé (completum) et un tout (totum). C'est en effet ainsi que nous définissons le tout, ce à quoi il ne manque rien, par exemple un homme total ou un coffre total. Et il en va du tout au sens éminent comme du tout particulier, à savoir c'est ce qui n'a rien à l'extérieur de soi. Ce à quoi il manque quelque chose qui existe hors de lui, cela n'est pas un tout, quoi que ce soit qui manque. Mais la totalité et l'achevé

natura tota in omnibus locis et temporibus et ubique et semper, quia perfecta et completa». Nous soulignons.

7. Voir par exemple Albert le Grand, Liber divisionum, tr. 2, c. 5, Alberti Magni Opera omnia, I/2, H. Möhle (éd.), Monasteri Westfalorum [Münster], Aschendorff, 2006, p. 91.

8. Boèce, De divisione liber, J. Magee (éd.), Leyde - Boston - Cologne, Brill, 1998, p. 12-14.

9. Ibid.: "dicitur quoque totum quod ex quibusdam virtutibus constat, ut animea alia potentia est sapiendi, alia sentiendi, alia vegetandi: partes sunt, sed non species».

10. Alexandre de Halès, Summa theologica, t. I, $\$ 71$, resp. ad arg. 2, p.111.

11. Aristote, Physica, Translatio vetus, in Aristoteles Latinus, t. VII-1, G. Verbeke (éd.), Leyde - New York, Brill, 1990, livre III, chap. 6, p.128, 1.3 (Bekker 207a): «Non enim cuius nichil est extra, sed cuius semper aliquid est extra, hoc infinitum est ». 
soit sont complètement la même chose, soit ont une nature voisine. Mais rien n'est achevé sans avoir de fin; or la fin est une limite ${ }^{12}$.

Il est aisé de voir que dans son deuxième texte sur l'immensité, Alexandre recourt aux formules mêmes de la traduction latine de la Physique (bien qu'Aristote ne soit pas explicitement cité ici). Il est non moins évident qu'Alexandre se sert de la définition aristotélicienne du tout, qui suppose une limite, pour penser la nature d'un être qui est précisément illimité. Visiblement, avec le totum perfectum, la perfection et l'infinité (prise cette fois en un sens déjà moderne) se sont rencontrées, ce qui rend possible une description conceptuelle de la présence d'immensité.

En effet, il ne faut pas perdre de vue l'objectif d'Alexandre: il n'a pas eu besoin de recourir au concept de totalité dans le traité sur l'essence et sur la simplicité; les concepts d'unité et de simplicité, ainsi qu'une forte sentence du Livre des Causes, la proposition 138, lui ont suffi ${ }^{13}$. Mais c'est au moment où il doit examiner le problème de la présence d'immensité, dans l'ordre du temps et de l'espace, que le besoin d'une meilleure compréhension de la totalité divine se fait jour.

On comprend alors pourquoi ce n'est pas Aristote, mais saint Anselme, qu'il s'attache à citer, très longuement. Alexandre cite deux passages remarquables du Monologion, où l'abbé du Bec essaie de montrer la cohérence de cette proposition apparemment contradictoire selon laquelle Dieu est tout entier partout et en tout temps, et en même temps n'est circonscrit par aucun lieu et par aucun temps. Il s'agit donc ici de penser le mode de présence de Dieu à ses créatures, et ce mode de présence n'est ni celui de l'infini aristotélicien (qui suppose un morcellement en parties et une incomplétude perpétuelle), ni exactement celui du tout aristotélicien (qui suppose une limite). La présence de Dieu tout entier à tout temps et en tout lieu devra donc être pensée comme pure et simple présence, sans composition, et sans limite:

Dans les choses, la même énonciation signifie deux choses, à savoir qu'elles sont présentes aux lieux et aux temps dans lesquels on dit qu'elles sont, et qu'elles sont contenues par eux; en l'essence suréminente au contraire, un

12. Ibid., 1. 4 sq. : "Cuius autem nichil est extra, hoc est perfectum et totum; sic enim diffinimus totum, cui nichil abest, ut hominem totum aut archam; sicut autem singulare, sic et quod est proprie, ut totum cuius nichil est extra; cuis autem absentia est extra, non omne est, cum absit. Totum autem et perfectum aut idem penitus omne aut proximum secundum naturam est; perfectum autem nullum non habet finem; finis autem terminus est».

13. «Toute puissance une est plus infinie qu'une puissance multiple», in La Demeure de l'être. Autour d'un anonyme, étude et traduction du Liber de Causis, P. Magnard, O. Boulnois, B. Pinchard et J.-L. Solère (trad. et éd.), Paris, Vrin, 1990, p. 65. 
seul sens est perçu, à savoir qu'elle est présente, non qu'elle est contenue. Si l'habitude de parler l'admettait, il semblerait par suite plus convenant de dire: elle est avec le lieu ou le temps, que: dans le lieu ou le temps ${ }^{14}$.

Et pour bien montrer que cette présence pure et impalpable n'en est pas moins une présence pleine et féconde pour la multiplicité des choses en présence, Anselme poursuit en disant: «elle est néanmoins d'une certaine manière en tout lieu ou temps, parce que tout ce qui est autre est soutenu par sa présence pour ne pas tomber dans le rien ${ }^{15}$.

Pour résumer cette seconde étape, on peut dire que malgré l'absence des concepts de simplicité et d'infinité, ce texte laisse surgir une figure du totum perfectum qui n'est compatible ni avec la composition ni avec la limite, mais qui ne perd pas pour autant sa possible proximité avec les choses multiples: au contraire, elle est ce qui leur donne d'être.

Dans la troisième étape de l'élaboration conceptuelle du totum, la source boécienne semble affleurer plus nettement. Le contexte est maintenant celui du traité de la puissance divine, au $\$ 151$. Il s'agit de répondre à la question : "qu'est-ce qui est absolument possible et impossible?». À un moment précis de sa réponse, le rédacteur cherche un exemple pour illustrer son propos et écrit: «il en va comme du concept de tout en géométrie comme tout continu (toto continuo), en logique comme tout universel (toto universali), et en métaphysique comme tout parfait (toto perfecto) ${ }^{16}$. L'apport de cette tripartition est qu'elle fait correspondre chaque niveau de signification à une discipline particulière: géométrie, logique, métaphysique. Mais il faut ajouter que les deux premières acceptions, tout continu et tout universel, ne sont pas tout à fait les mêmes que précédemment, et que l'affluent aristotélicien n'est pas prioritaire. Cette fois, la source la plus importante est probablement le De divisione de Boèce (qui n'est pas cité explicitement). Boèce propose en effet une analyse du totum où il est aisé de retrouver l'exposé halésien: le totum désigne d'abord le continu, comme le corps ou la ligne. En un second sens (qui n'apparaît pas dans la Summa), le tout

14. Anselme de Cantorbéry, Monologion, chap. 22, in L'Euvre de saint Anselme de Cantorbéry, t. I, M. Corbin (éd.), Paris, Cerf, 1986, p. 40: «In illis namque duo quaedam eadem prolatio significat, id est: quia et praesentia sunt locis et temporibus in quibus esse dicuntur, et quia continentur ab ipsis; in summo vero essentia unum tantum percipitur, id est; quia praesens est, non etiam quia continetur. Unde si usus loquendi admitteret, convenientius dici videretur esse cum loco vel tempora quam in loco vel tempore».

15. Ibid., p. 41: «et tamen in omni loco vel tempore suo quodam modo dici potest esse, quoniam quidquid aliud est ne in nihilum cadat ab ea praesente sustinetur".

16. Alexandre de Halès, Summa theologica, t. I, \$151, resp., p. 231: «sicut intentio in geometricis pro toto continuo, in logicis pro toto universali, in metaphysicis pro toto perfecto". 
désigne un ensemble discontinu (par exemple un peuple). En un troisième sens, le tout désigne l'universel (par exemple l'homme ou le cheval), qui se prédique de ses parties de manière égale (les hommes ou les chevaux particuliers, qui ne sont pas plus ou moins hommes ou chevaux). Enfin, en un quatrième sens, le tout est ce qui consiste en plusieurs vertus ou puissances, et l'exemple choisi par Boèce est celui de l'âme, en qui diffèrent la puissance de connaître, la puissance de sentir et la puissance végétative (nous reconnaissons le totum virtuale) ${ }^{17}$.

De nouveau, la question se pose: quel est le rapport entre cette dernière acception et le totum perfectum qui prend sa place dans l'exposé d'Alexandre? Peut-on dire qu'il y a une sorte de confusion entre le totum perfectum et le totum de puissance? Cela n'est pas impossible, puisque dans les deux cas émerge une figure du tout où la multiplicité ne partitionne pas le tout, mais en exprime la complétude. Il faudrait également déterminer ce qu'Alexandre veut dire lorsqu'il attribue le totum perfectum à la métaphysique. S'agit-il de la science de l'être divin, «la théologie des philosophes » comme la dénomme Alexandre ${ }^{18}$ ? Dans ce cas, l'application du concept de totum perfectum à l'Éternel serait en quelque sorte légitimée et confirmée. Mais il n'est pas exclu que par ricochet, le totum perfectum ne soit pas un concept utile pour penser l'âme ou le monde. Ce qui est certain en tout cas, est que son libre usage des sources disponibles a pour but de construire un concept de tout qui soit compatible à la fois avec la perfection et l'infinité divines (qui impliquent l'absence de partition) et avec une proximité effective vis-à-vis des choses singulières. En ce sens, le totum virtuale est évidemment un modèle insuffisant et inapplicable à Dieu, mais il est incontestablement supérieur aux autres formes du tout; aussi peut-on penser qu'à ce titre, dans l'esprit des rédacteurs de la Summa Halensis, il est une espèce du genre totum perfectum, bien qu'aucun texte ne le présente explicitement comme tel.

La question de la présence d'immensité n'est pas le seul vecteur de réflexion sur le totum. Le livre II (lieu habituel des questions sur le monde) fournit aussi l'occasion de revenir sur cette notion et sur ses potentialités. Cette fois, c'est le monde comme tout qui est en question. Bien sûr, il ne s'agit pas de savoir si le monde est composé ou non, s'il est un tout intégral ou non - car il l'est, de toute évidence; ce qui vaut pour la maison prise en exemple par Boèce pour illustrer le tout intégral vaut certainement pour le monde dans son ensemble. En revanche, ce qui vaut la peine d'être examiné

17. Boèce, De divisione liber, p. 12-14.

18. Voir Summa theologica, t. I, tract. introductorius, $\$ 1$, p. 2. Sur ce point, voir Philosophie et théologie au Moyen Âge, t. II, O. Boulnois (dir.), Paris, Cerf, 2009, p. 193 sq. 
est la question de savoir s'il n'est pas un peu plus qu'un tout intégral, et même, s'il n'est pas lui aussi une sorte de totum perfectum. Pour défendre cette thèse, il suffirait par exemple de réinvestir la thèse néoplatonicienne de l'âme du monde. D’où la question posée au $\$ 96$ : utrum anima mundi sit perfectio universi? Nous avons vu avec Boèce que l'âme immanente à ses puissances (et à son corps) fournit un modèle important pour penser l'unité simultanément présente en tout lieu et en quelque sorte antérieure à ses parties. Ne peut-on pas dire, en ce sens, que le monde est un tout non seulement intégral, mais potentiel, non seulement organisé, mais organique, pour reprendre la distinction kantienne de la Critique de la faculté de juger ${ }^{19}$ ?

Alexandre ne peut pas renoncer à l'idée selon laquelle le monde, comme megacosmus ou macrocosmos, selon les deux termes qu'il utilise, est un tout parfait, comme l'est à sa façon chaque vivant en particulier, et l'homme d'une manière éminente. Sur ce point, les auteurs franciscains (voire médiévaux en général) inscrivent l'articulation de leur cosmologie et de leur anthropologie dans la continuité des réflexions platoniciennes du Timée, et dans celle de la pensée stoïcienne, tout en l'enrichissant de correspondances symboliques. Alexandre doit néanmoins tenir compte des mises en garde d'Augustin par rapport à la thèse de l'âme du monde ${ }^{20}$. Il distingue alors (outre le totum perfectum divin) deux types de perfection, c'est-à-dire deux types de réalisation d'unité. Dans le premier, le principe d'unité fait un avec les parties qu'il réalise (nous discernons derrière cette définition le modèle du vivant, de l'être animé): «l'âme est la perfection qui fait un avec son perfectible, non seulement comme le moteur avec ce qu'il meut, mais comme la forme substantielle avec le sujet qu'elle parfait ${ }^{21}$. C'est du bon aristotélisme: l'âme ne fait pas seulement fonctionner le corps, elle le fait être. Dans le second cas, la perfection ne tient pas à la présence d'une âme, mais simplement à la présence d'un moteur, et suivant la situation de ce moteur par rapport à ce qu'il meut, on parlera de «perfection externe» ou

19. Kant, Critique de la faculté de juger, A. Renaut (trad.), $2^{\mathrm{e}}$ éd., Paris, Flammarion (GF, 1088), $1995, \$ 65$, p. 364-368.

20. Augustin, La Cité de Dieu, IV, 12, in Euvres, t. II, L. Jerphagnon (éd.), Paris, Gallimard (Bibliothèque de la Pléiade, 468), 200o, p. 149: «Le problème suivant ne doit-il pas émouvoir les hommes intelligents, ou plutôt tous les hommes quels qu'ils soient [...]: si Dieu est l'Âme du monde et le monde le corps de Dieu, et s'il en résulte un unique être vivant composé d'âme et de corps; que ce Dieu, sein de la nature, contienne toutes choses en lui, de sorte que de son âme, principe vivifiant de toute cette machine, découlent la vie et l'âme de tous les vivants d'après le sort échu à chacun à la naissance, il ne reste plus rien qui ne soit une partie de Dieu. S'il en est ainsi, qui ne voit quelle impiété, quelle irréligion en résultent».

21. Summa theologica, t. II, $\$ 96$, p. 118 : «anima est perfectio, quae facit unum cum suo perfectibili, non tantum sicut motor cum mobili, sed sicut forma substantialis cum subiecto quod perficit». 
de «perfection interne». Pour ce qui est du monde, la perfection interne est liée à «la nature du premier ciel, à laquelle sont subordonnées les natures inférieures par l'influence d'un mouvement »; dans cette perfection interne, le principe d'unité est l'un des éléments, l'une des parties de l'ensemble. Quant à la perfection externe, elle désigne «la vertu séparée qui meut le ciel», et qui n'est pas pour autant le premier moteur. Cette vertu séparée, c'est l'action des anges "qui ornent et administrent l'univers ${ }^{22}$.

Par conséquent, on peut bien dire que l'univers ou le monde est un tout parfait, mais non au sens de l'âme unie au corps et non au point d'être ce que Proclus ou Denys appellent une totalité, partout présente, sans partie, et imparticipable. À l'âme du monde, les rédacteurs de la Summa substituent donc une double perfection motrice, ce qui leur permet de dépasser une conception simplement intégrative de l'univers, sans tomber pour autant dans les embarras d'une âme du monde.

Bien qu'Alexandre ne juge pas utile d'employer la notion de totalitas, il se trouve néanmoins dans l'obligation de distinguer différentes sortes de "tout parfait», qui sont définis à partir d'Aristote (Physique III), d'Anselme (Monologion), et du De divisione de Boèce: (1) le tout parfait qu'est Dieu, en son essence: unité sans partie, qui n'entre dans la composition d'aucune autre chose, absolument simple et présente à toute chose; (2) le tout parfait du vivant, en tant que son âme est l'unité qui réalise son corps, et en tant que cette âme est l'unité de ses puissances; (3) le tout parfait qu'est l'univers, en tant qu'il est mû par un seul principe interne et par une puissance externe.

Au moment où l'aventure de la Summa Halensis s'arrête (1245) et où Bonaventure est sur le point de prendre la relève de cette réflexion par la rédaction de son propre Commentaire des sentences, deux tâches doivent encore être menées: premièrement, la jonction des questions relatives à la simplicité avec le concept de totum perfectum, appliqué à Dieu dans le contexte de la présence d'immensité; deuxièmement, une articulation plus nette non seulement ornementale, mais métaphysiquement fondée,

22. Ibid., p.119: "non est mundus animal nec perfectio eius est anima, sed habet duplicem perfectionem: unam intra, et haec dicitur esse natura primi caeli ad quam ordinantur aliae naturae inferiores per quamdam influentiam motus, et haec perfectio est intra; est autem alia perefectio extra, quae tenet rationem motoris separati [...]. Per quod designatur quod est quaedam virtus separata quae proximo movet caelum; et quia est ad ministrandum, non est illa virtus divina, quae tamen virtus divina per illam tamquam ministram movet. Nulla ergo istarum perfectionum dicetur esse anima; non enim natura ipsius mobilis recte dicitur anima: anima enim est motor distinctus per essentiam a corpore mobile, natura vero illa non est distincta per essentiam a mobile. Neque virtus illa spiritualis, de qua logitur Augustinus [Retractationes, I, 11, 4], quae est in angelis, non recte dicetur anima mundi». Sur la fonction cosmologique des anges, voir T. Suarez-Nani, Les Anges et la philosophie, Paris, Vrin, 2002, p. 91-190. 
des différents niveaux de totalités à partir de Dieu lui-même. Le premier point étant amplement traité dans l'article de Chirine Raveton, il ne sera ici question que du second.

\section{L'ordre expressionniste des totalités chez saint Bonaventure}

Bonaventure, contrairement aux rédacteurs de la Summa Halensis, utilise le mot totalitas. Est-ce le signe d'une prise de conscience de sa part, d'une volonté de distinguer absolument totum et totalitas? Ce serait beaucoup dire. Il semble plutôt, à première vue, que le mot totalitas soit chez lui l'équivalent du totum perfectum. On peut alléguer au moins deux textes montrant le lien entre totalitas et totum perfectum. Citons d'abord la cinquième Question disputée sur le Mystère de la Trinité, qui présente justement une réflexion sur la présence d'immensité:

[L]a totalité, que l'on pose dans l'éternité, ne doit pas être entendue selon la quantité de masse, mais selon la quantité de vertu, dans laquelle se tient la raison de grandeur et d'immensité avec la perfection de la simplicité [...] "total et simultané» se dit ici plutôt selon la raison de perfection que selon la raison de distribution dans les parties d'un tout intégral ${ }^{23}$.

Il est clairement question de la totalité divine, conçue par contraste avec le tout intégral. Dans un cas, la totalité est associée à la perfection, dans l'autre, à la distribution et à la division. La totalité est donc pensée par distinction avec le tout comme composé de parties, et en relation étroite avec les concepts de perfection, d'immensité et de simplicité. Il est clair qu'en ce sens, sa physionomie est plus précise que le totum perfectum d'Alexandre, qui n'articulait pas explicitement simplicité, perfection et unité.

Un deuxième texte confirme cette analyse: dans le Commentaire des sentences, premier livre, distinction 19, suivant le plan des Sentences de Pierre Lombard, Bonaventure pose deux questions qui concernent le tout: d'abord, faut-il poser en Dieu un tout intégral? Puis, faut-il poser en Dieu un tout universel? Dans les deux cas, la réponse est évidemment négative. Or la première question est l'occasion d'un court exposé sur le tout:

23. Bonaventure, Quaestiones disputatae de mysterio Trinitatis, Quaestio V, art. 1, resp. ad arg. 9, in Opera omnia, t. V, Quaracchi, Ad Claras Aquas, 1891, p.91: «totalitas, quae ponitur in aeternitate, non attenditur secundum quantitatem molis, sed secundum quantitatem virtutis, in qua simul ratio magnitudinis et immensitatis cum perfectione simplicitatis [...] totum simul dicitur ibi potius secundum rationem perfectionis quam secundum rationem distributionis in partes alicuius totius integralis». 
En un premier sens, le tout ou totalité se dit de manière absolue; et de cette manière, le tout est la même chose que le parfait. En un autre sens, le tout se dit par rapport à la partie; et en ce sens, on appelle «tout» ce qui a des parties. Au premier sens, il est bien de poser la totalité en Dieu, mais non au second sens, de même que le fait d'être composé de partie ${ }^{24}$.

De cette permission accordée pour appeler Dieu «totalité» et admettre ce concept comme un nom divin ou en tout cas un nom philosophique de Dieu (ce qui n'était pas arrivé, semble-t-il, depuis Les Noms divins de Denys), découlent deux consignes linguistiques précises dans la distinction 37 : totalitas peut être utilisé comme un synonyme de simplicité ou comme un synonyme d'infinité ${ }^{25}$; et dans ce dernier cas, précise Bonaventure, on utilisera plutôt l'adverbe totaliter, comme dans l'expression totus sed non totaliter pour parler de la connaissance des bienheureux ${ }^{26}$. Au final, totalitas apparaît donc comme une autre manière de parler de la perfection divine, qui est elle aussi un concept médian entre simplicité et infinité.

D'un côté, nous obtenons donc un concept aux contours moins flous que le totum perfectum du premier livre de la Summa Halensis. Mais ce que nous gagnons en détermination du côté de la théologie s'accompagne paradoxalement d'une nouvelle extension du concept et donc d'une possible dissolution de sa signification: pourquoi Bonaventure fait-il rejaillir le sens divin de la totalité sur le monde et sur l'homme? Là encore, il y a deux manières d'interpréter ce transfert: soit on y voit le signe que la totalité n'est pas un concept aussi déterminé que cela, et qu'il est trop souple dans sa signification pour être véritablement utile; soit on considère que ce transfert de totalité est cohérent et signifiant.

En ce qui concerne le monde, prenons l'exemple de la deuxième partie du Breviloquium. Le titre du premier chapitre n'est autre que «De productione mundi totalis», "Au sujet de la production du monde total». Manifestement, on peut y lire une volonté de présenter le monde comme un tout organisé, fermement structuré par sa dualité ciel/terre, invisible/visible, comme

24. Bonaventure, I Sent., d. 19, p. II, q. 1, resp., in Opera omnia, t. I, Quaracchi, Ad Claras Aquas, 1882, p. 356: «Totum sive totalitas uno modo dicitur absolute: et sic totum idem est quod perfectum. Alio modo dicitur totum per comparationem ad partem: et sic dicitur totum quod habet partem et partem sive partes. Primo modo bene est ponere totalitatem in Deo, secundo non, nec etiam partialitatem ».

25. Ibid., d. 37, p. II, a. 1, q. 1, resp. ad arg. 4, p. 653: «Quando ergo apponitur circa Deum nomen importans totalitem, potest dupliciter apponi: vel ratione simplicitatis; et sic privat partem et partem, et sic significatur per nomen, ut si dicatur: totus Deus. [...] Potest iterum totalitas apponi ratione infinitatis et hoc fit per adverbium, ut si dicatur: Deus est totaliter in loco hoc sive secundum totum; et sic, cum divina infinitas a nullo loco includatur, Deus in nullo est secundum totum".

26. Voir par exemple Bonaventure, I Sent., d. 3, p. I, a. un., resp. ad arg. 3, t. I, p. 69. 
l'indique le plan de l'ouvrage. Mais on peut penser qu'il y a une raison plus profonde à cela; ce «monde total» est en effet l'expression de la totalité divine: «la créature du monde est comme un livre dans lequel éclate, est représentée et est lue la Trinité fabricatrice, selon trois degrés d'expression, à savoir sur le mode du vestige, de l'image et de la ressemblance ${ }^{27}$. Le Dieu unique, parfait, simple et infini, se manifeste dans un monde unique, qui n'est certes ni simple ni infini, mais qui, en tant qu'expression et révélation de la totalité divine, peut bien être appelé «total». Et ce qui est vrai du monde comme tel l'est aussi de chaque créature prise à part: dans sa condition de vestige (qui est universelle ${ }^{28}$ ), chaque créature est le miroir de la totalité divine, soit considérée comme essence, soit considérée en tant qu'elle est possédée par les personnes. Ainsi, chaque vestige exprime à la fois l'unité, la vérité et la bonté divine (on reconnaît les transcendantaux), mais on peut également y contempler les trois personnes divines. Or Bonaventure semble être le seul auteur du XIII ${ }^{\mathrm{e}}$ siècle à avoir défendu la thèse d'une représentation non pas partielle, mais totale de Dieu dans le vestige - représentation éloignée, si l'on veut, mais totale, l'idée même de représentation partielle de Dieu n'étant pas compatible avec sa simplicité29. Bien sûr, par rapport à Dieu, un vestige n'est pas une totalité, étant un être composé ou entrant dans la composition d'un autre. Mais du point de vue de l'expression, il est aussi plus qu'un tout composé de parties et bien organisé, précisément parce qu'il exprime, sans y participer, la simplicité et la perfection de son principe.

Un rapide coup d'œil au livre II du Commentaire des sentences nous permettra de vérifier cette position et de voir comment cette totalité sensible qu'est le monde s'articule avec la totalité divine et avec ce que Bonaventure appelle la totalité spirituelle. Dans la distinction 16, la première question du premier article concerne la notion d'image de Dieu : en quel sens peuton dire que l'homme, conformément au verset de la Genèse, est image de

27. Bonaventure, Breviloquium, II, 12, $\$ 1$, in Opera omnia, t. V, Quaracchi, Ad Claras Aquas, 1891, p. 30 : «creatura mundi est quasi quidam liber, in quo relucet, repraesentatur et legitur Trinitas fabricatrix secundum triplicem gradum expressionis, scilicet per modum vestigii, imaginis et similitudinis».

28. Ibid.: «ratio vestigii reperitur in omnibus creaturis».

29. Bonaventure, I Sent., d. 3, p. I, q. 2, resp. ad arg. 4, in Opera omnia, t. I, p.73: «Ad illud quod ultimo quaeritur de differentia vestigii et imagini, quidam assignant, quod vestigium est in sensibilibus, imago in spiritualibus. Sed ista distinctio et positio non valet, quia vestigium est etiam in spiritualibus. Nam unitas, veritas, bonitas, in quibus consistit vestigium, sunt conditiones maxime universales et intelligibiles. Alii dicunt, quod vestigium dicitur, quia repraesentat secundum partem, sed imago secundum totum. Sed haec iterum differentia non valet, quia, cum Deus sit simplex, non habet repraesententans secundum partem; cum iterum sit infinitus, a nulla omnino creatura, etiam a toto mundo non potest repraesentari secundum totum». 
Dieu? D'après l'un des arguments opposés à la thèse défendue, l'homme ne peut pas être une telle image, mais seulement un vestige: étant un être fini, il ne peut représenter Dieu que partiellement, alors que l'image a pour caractéristique de représenter selon le tout; or représenter Dieu selon le tout, suppose d'être soi-même infini. Voici la réponse de Bonaventure à cet argument:

À l'objection selon laquelle l'image représente selon le tout, il faut répondre que cela est vrai de cette image qui possède une perfection absolue; et en ce sens, seul le Fils de Dieu est l'image du Père. De l'autre [image], il n'est pas vrai qu'elle représente selon le tout absolument, mais elle représente selon une certaine totalités ${ }^{\circ}$.

Outre qu'il considère qu'il n'existe pas de représentation partielle de Dieu, même dans le vestige, Bonaventure distingue ici deux sortes d'image, et la première d'entre elles n'est autre que le Fils de Dieu, image parfaite et infinie du Père. Non pas que cette image représente toutes les parties qui composeraient le Père; au contraire, dans la génération éternelle, c'est l'essence divine comme totalité simple, parfaite et infinie qui est donnée au Fils. Donc, si être l'image de Dieu consiste à recevoir la totalité divine en plénitude, seul le Fils, effectivement, peut être dit «image de Dieu», et donc ressemblance totale, à la fois imitative et expressive ${ }^{31}$. Cependant, Bonaventure ménage "en dessous» de cette totalité (cette fois exprimée de manière trinitaire) une place pour une certaine totalité dans l'image humaine: cette image-là ne représente pas Dieu secundum totum simpliciter, selon le tout absolument, mais quantum ad aliquam totalitatam, selon une certaine totalité. La structure de dérivation d'une totalité à l'autre est très visible ici: c'est bien à partir de la totalité première qu'est définie la seconde, qui prend alors un sens atténué, mais réel. Qu'est-ce qui justifie une telle dérivation, dans le cas de l'homme? Bonaventure l'explique ensuite:

En effet, comme «la créature rationnelle, ou l'intellect, est en quelque sorte toutes choses» [De anima, III, 8], toutes choses sont destinées à y être inscrites, et les ressemblances de toutes choses à y être imprimées et peintes. C’est

30. Bonaventure, II Sent., d. 16, a. 1, q. 1, resp. ad arg. 5., in Opera omnia, t. II, Quaracchi, Ad Claras Aquas, 1885, p. 395-396: «Ad illud quod obiicitur, quod imago repraesentat secundum totum; dicendum quod illud est verum de illa imagine, quae habet omnimodam perfectionem; et sic solus Filius Dei est imago Patris. De alia non est verum, quod repraesentet secundum totum simpliciter, repraesentat autem quantum ad aliquam totalitatem».

31. Bonaventure, I Sent., d. 27, p. II, a. 1, q. 2, in Opera omnia, t. I, p. 485: «[C]oncipit sive generat unum Verbum, quod est similitudo Patris imitativa et similitudo rerum exemplativa et similitudo operative; et ita tenet quasi medium, et dicitur Pater operari per Verbum; et ulterius ipisi Verbo atribuitur, quod sit Dei virtus et Dei sapientia». 
pourquoi, de même que tout l'univers représente Dieu selon une totalité sensible, de même la créature rationnelle le représente selon une totalité spirituelle ${ }^{32}$.

L'argumentation est assez complexe: Bonaventure justifie le fait que l'homme, comme créature rationnelle, représente Dieu selon une certaine totalité par le fait que l'homme représente le monde. Plus exactement, on peut dire que l'homme exprime Dieu sur le mode de la totalité, parce que l'univers entier s'imprime en lui. Manifestement, il manque une médiation afin de comprendre pourquoi l'impression de l'un vaut pour expression de l'autre. Cette médiation manquante, c'est le Verbe: celui pour qui exprimer Dieu et exprimer le monde sont une seule et même chose, une seule et même vie, c'est précisément le Fils, lui que Guillaume d'Auxerre, dans la Summa aurea, appelait "monde archétype " ${ }^{33}$. Le fait est que Bonaventure n'utilise pas cette expression, qui projette sur le Fils le modèle du tout intégral qu'est le monde, en même temps que celui du «tout universel», à travers la notion d'archétype. Dans ce cas, comment comprendre que le Fils soit l'expression parfaite de la totalité divine comme de la totalité du monde, sans devenir pour autant un simple tout? Il est nécessaire de retravailler en profondeur la théorie des idées ${ }^{34}$. C'est ainsi que Bonaventure ne perd jamais une occasion de montrer que l'hospitalité du Fils à l'égard de la multiplicité ne relève pas d'une pré-contenance ou d'une intégration, mais de l'expression absolument simple de ce que le Père veut et fait. Deux précautions assez significatives de sa part doivent être évoquées : d'une part, le refus du concept de participation ${ }^{35}$, d'autre part, l'indépendance du Fils par rapport au genre, à l'universel et au particulier. La distinction $35 \mathrm{du}$ premier livre du Commentaire des sentences est très claire à ce sujet, et ses conclusions seront reprises et approfondies quelque temps plus tard dans la troisième des Questions disputées sur la science du Christ ${ }^{36}$ :

32. Bonaventure, II Sent., d. 16, a. 1, q. 1, resp. ad arg. 5, in Opera omnia, t. II, p. 395-396: «Quia enim "rationalis creatura et intellectus quodam modo est omnia", et omnia sunt nata ibi scribi, et imprimi omnium similitudines et depingi; ideo, sicut totum universum repraesentat Deum in quadam totalitate sensibili, sic creatura rationalis eum repraesentat in quadam totalitate spirituali».

33. Guillaume d'Auxerre, Summa aurea, J. Ribaillier (éd.), Paris, CNRS - Grottaferrata, Ad Claras Aquas (Spicilegium Bonaventurianum, 16), 1980.

34. L. Solignac, La Voie de la ressemblance, Paris, Hermann, 2014, p.168-196.

35. Par exemple Bonaventure, I Sent., d. 35, q. 1, resp. ad arg. 2, in Opera omnia, t. I, p. 601.

36. Bonaventure, Quaestiones disputatae de scientia Christi, q. III, resp., in Opera omnia, t. V, p. 14 : «comme la vérité en tant que telle se comporte indifféremment vis-à-vis de toutes choses, et que toutes les autres choses sont pour elle matérielles, elle peut être, en tant qu'acte pur, la ressemblance expressive de toutes choses» (nous soulignons). 
C'est pourquoi il faut dire qu'il y a une ressemblance qui est selon la propriété $d u$ genre; et il n'y a pas de doute à son sujet: elle ne peut pas être unique pour les nombreuses choses qui diffèrent par le genre; et il s'agit d'une ressemblance qui est exprimée et causée par une chose d'un genre déterminé. Autre est la ressemblance qui est absolument hors genre; elle est ainsi parce qu'elle n'est pas astreinte à un genre: ni en raison de ce qu'est ceci ou de ce qu'est cela, ni en raison de ce qu'il est selon la partie ou selon le tout; et une telle ressemblance est la vérité divine et l'idée en Dieu ${ }^{37}$.

Paradoxalement, cette indépendance radicale du Verbe à l'égard des créatures permet une proximité maximale avec elle. Le Verbe est ubique, partout, par sa génération éternelle ${ }^{38}$, mais il n'est partout que par sa "présence seule» dit encore Bonaventure ${ }^{39}$, retrouvant ainsi l'intuition d'Anselme. Ainsi exprime-t-il le monde parce qu'il exprime le Père, ainsi est-il présent dans le monde, parce qu'il est éternellement engendré par le Père.

Pour finir, il convient de revenir sur la nature de l'ordre qui réunit et distingue à la fois ces différentes totalités. Pour commencer, nous pouvons proposer l'hypothèse suivante, d'inspiration gilsonnienne ${ }^{40}$ : il s'agit d'une dérivation analogique à partir de la totalitas divine - laquelle se caractérise par sa simplicité, son infinité, sa perfection, son indépendance parfaite à l'égard de toutes choses, et donc par son omniprésence. À partir de cette unité focale, la totalité se décline sur deux niveaux: celui de la totalité spirituelle, par laquelle l'âme humaine prend en charge dans son unité et sa perfection propres la multiplicité des choses, et celui de la totalité sensible qui, en tant que vestige, représente Dieu non pas partiellement,

37. Bonaventure, I Sent., d. 35, q. 2, resp. ad arg. 2, in Opera omnia, t. I, p. 606: «Propter hoc dicendum quod similitudo quaedam est secundum proprietatem generis; et de hac non est dubium, quod non potest esse una plurium genere differentium; et haec est similitudo, quae exprimitur et causatur a re generis determinati. Alia est similitudo simpliciter extra genus; et haec, quia ad hoc genus non arctatur, qua ratione est huius, ea ratione est illius, et qua ratione est huius secundum partem, eadem ratione secundum totum; et talis similitudo est divina veritas et idea in Deo".

38. Bonaventure, Itinerarium mentis in Deum, II, 7 , in Opera omnia, t. V, p.301.

39. Bonaventure, I Sent., d. 37, p. I, a. 1, q. 1, resp. ad arg. 1, ibid., p. 639 : «il y a trois façons pour une chose d'être unie à une autre : soit selon la présence seule, soit selon la présence et la dépendance, soit selon la présence, la dépendance et la concomitance dans la matière. C'est de la première façon que le rayon [lumineux] est dans l'air, c'est de la seconde façon que l'âme est dans le corps, et c'est de la troisième façon que le liquide est dans le liquide. Ce qui est dans quelque chose selon le premier mode n'y est pas mêlé, de même ce qui y est selon le second mode n'y est pas proprement mêlé, mais seulement ce qui y est selon le troisième. Et c'est selon le premier mode que Dieu est dans les choses, et non selon le deuxième ou le troisième».

40. É. Gilson, La Philosophie de saint Bonaventure, $2^{\mathrm{e}}$ éd., Paris, Vrin, 1953, p.165-191. 
mais totalement. Cette hypothèse doit toutefois être complétée, car elle ne prend pas en compte la fonction spécifique du Verbe dans cette dérivation.

Essayons donc une deuxième hypothèse: n'avons-nous pas affaire, tout simplement, à une reprise de la théorie néoplatonicienne de l'émanation, où des unités de plus en plus composées et divisées se diffusent les unes à partir des autres? C'est d'ailleurs ce schéma qui est repris dans le Livre des Causes, encore attribué à Aristote par nos auteurs et assez souvent cité dans leurs ouvrages: l'unité la plus simple et la plus puissante produit l'âme, qui est une unité moins simple, sujette à la division, laquelle produit à son tour ces unités très divisées et affaiblies que sont les corps ${ }^{41}$. Nous retrouvons ainsi les trois niveaux, divin, spirituel et sensible. Cette hypothèse est intéressante, mais elle ne tient pas vraiment compte des textes cités précédemment: l'objectif du Docteur séraphique est de conserver le maximum de multiplicité dans l'unité première et divine, par la vie trinitaire et son expression dans le Verbe, et le maximum d'unité dans les créatures les plus humbles, par l'extension du paradigme trinitaire à toute chose. En outre, la totalité spirituelle prend place non pas en amont du monde, comme dans l'émanation décrite par le Livre des Causes, mais en aval, ou encore, non pas dans le processus de l'exitus, mais uniquement dans celui de la reductio (reconduction) du monde sensible vers le Père. De ce fait, la structure d'émanation, ayant subi des modifications aussi importantes (et sans même parler de la différence fondamentale entre émanation et création), ne peut pas permettre d'appréhender adéquatement l'ordre bonaventurien des totalités.

Examinons alors une dernière hypothèse en vue de décrire la structure d'articulation des trois totalités: il s'agit de l'hypothèse monadologique proposée par Hans Urs von Balthasar, reprise par les commentateurs français, et selon laquelle cet ordre hiérarchique des totalités forme chez Bonaventure une monadologie, c'est-à-dire un système de monades qui $s^{\prime}$ expriment les unes les autres ${ }^{42}$. La grande force de cette interprétation est la prise en compte de la fonction spécifique du Verbe dans ce dispositif d'expressionnisme total. En ce sens, elle pallie le défaut de l'hypothèse analogique. Toutefois, une réserve s'impose: il n'y a précisément pas de «supertotalité», chez Bonaventure, qui pourrait porter le nom de monadologie,

41. La Demeure de l'être..., chap. XIII, prop. 115-117, p. 61: «Les choses sensibles sont en toute âme parce qu'elle en est le modèle, et les choses intelligibles sont en elle parce qu'elle les connaît. / Et il n'en est ainsi que parce qu'elle est intermédiaire entre les choses intelligibles, qui ne sont pas mues, et les choses sensibles qui sont mues. / Et parce qu'ainsi est l'âme, il se fait qu'elle façonne les choses corporelles: pour cette raison, elle se trouve être cause des corps, et causée à partir de l'intelligence, qui est avant elle».

42. H. U. von Balthasar, La Gloire et la Croix, Paris, Aubier, 1968, t. II, p. 304. 
c'est-à-dire, au fond, une totalité qui comprendrait toutes les autres à titre de parties, à la manière d'un tout intégral. De ce fait, il n'existe pas non plus de procédé spéculatif par lequel on pourrait quitter son point de vue de créature. En revanche, le Frère mineur propose une nouvelle définition de la métaphysique, dans Les Conférences sur les six jours de la création, comme science des relations d'impression et d'expression mutuelles entre les êtres ${ }^{43}$ et comme art de fabriquer des miroirs, des petits instruments spéculatifs, au sens littéral du terme, pour prendre sur le fait la présence divine dans les créatures ${ }^{44}$. L'attention du métaphysicien au singulier sera donc ici plus cruciale que l'attention à l'universel. Sans doute faut-il y voir un effet concret et tardif, une traduction philosophique, si l'on veut, du dogme de l'Incarnation. La totalité divine ne pourra donc être appréhendée (à défaut d'être comprise) que dans la pauvreté d'une unité insuffisante et composée, voire dans le miroir si paradoxal de la Croix où se démet et se disloque celui qui est absolument simple. De fait, comme l'écrit Bonaventure dans la huitième conférence de l'Hexaëmeron, «l'humilité de Dieu fait homme est si grande que la raison défaille ${ }^{45}$.

Laure SolignaC

Institut catholique de Paris Faculté de philosophie, UR «Religion, culture et société», EA 7403

43. Bonaventure, Collationes in Hexaëmeron, I, 13, in Opera omnia, t. V, p. 331: «Sed ut considerat illud esse in rationeomnia exemplantis, cum nullo communicat et verus est metaphysicus».

44. Sur la fabrique des miroirs, L. Solignac, La Voie de la ressemblance, p. 430-434.

45. Bonaventure, Collationes in Hexaëmeron, VIII, 5 , in Opera omnia, t. V, p. $370:$ :Profunditas Dei humanati, scilicet humilitas, tanta est, quod ratio deficit». 
